\title{
THE OBSERVATION OF COUNTERJETS OF QUASARS AND GALAXIES
}

\author{
S. M. GONG (KUNG) \\ Purple Mountain Astronomical Observatory \\ Chinese Academy of Sciences \\ 2 West Beijing Rd., Nanjing 210008, China
}

\begin{abstract}
The purpose of this paper is to point out the importance of the observation of the counterjet of subluminals, for it will settle up the question whether quasars redshifts are cosmological or not. If so, from the observed wavelength of the counterjet, one is able to find out the value of Hubble constant $H_{0}$. What we observe as an apparent superluminal or subluminal motion is the resultant velocity of Hubble flow and a local ejection. Taking Hubble flow into account, one can explain problems posed for the relativistic beaming model, such as the one-sidedness problem and the untolerable extension of the deprojected structure of some superluminal sources. Our method is applied to two superluminals, 4C.73.18 and $3 \mathrm{C} 179$, and two subluminals, M87 and 3C.84. Our method can also be applied to investigate the problem of the ejection of the quasar from the galaxy in the framework of kinematics. Two quasar-galaxy pairs, Markarian 205-NGC4319 and 3C455-NGC7413, are studied.
\end{abstract}

\section{INTRODUCTION}

In the past few years a great progress has been made in the VLBI images, such as the "WORLD ARRAY" images of M87 made by Biretta and Owen (1990) and 3C48 obtained by Wilkinson et al.(1990), showing structural complexity that rivals that seen in VLA. However, the theory to interpret these valuable data lags behind. The most generally accepted explanation appears to be the relativistic beaming model, first suggested by Rees $(1966,1967)$ and elaborated for relativistic jet by Behr et al. (1976) and Blandford and Konigl (1979). Problems posed for the relativistic model, such as the one-sidedness problem and the uncommonly largely intrinsic size of some superluminal sources, are still challenging current workers at the recent workshops (Superluminal Radio Sources, 1987; Parsec-scale Radio Jet, 1990) and IAU Symposium 129 (Impact of VLBI on Astrophysics and Geophysics, 1988).

Rees' formula, $v_{a p p}=v \sin \theta /(1-v \cos \theta)($ the velocity of light $c=1)$, relates the apparent transverse velocity $v_{a p p}$ with the ejection velocity $v$, where $\theta$ is the angle between $v$ and the line of sight. Rees' formula is good only for the ejecting body, relatively at rest with respect 
to an observer. Now the ejecting body, a quasar, usually has large receding velocity. We have to take the velocity of Hubble flow into consideration.

In the literature a few works (Dishon \& Weber, 1977; Horak, 1978; Li, 1980) tried to solve the problem of superluminal motion with the consideration of Hubble flow. In addition to the unsound assumption about the quasar to have the transverse velocity of the ejecta in the first two works and the introduction of unconfirmed proper motions of quasars in the third, all three works said nothing about the radial component of the velocity of the ejecta. They had not really solved the problem, nevertheless, it is helpful to point out the need of the consideration of Hubble flow.

In the simple case of a quasar ejecting materials in the direction of line of sight, Balicall et al. (1967) and Weymann et al. (197T) had successively derived the relative velocity, $v_{r \in l}$ , between the absorption and emission regions by taking the velocity of Hubble flow into account. The relative velocity is no other than the velocity of local ejection seen from the quasar. An observer on the Earth will see velocity of the absorption material, not the velocity of local ejection.

\section{THE SOLUTION FOR THE OBSERVATION OF COUNTERJET OF QUASAR}

The solution of the subluminal motion is the same as that of the superluminal one which we have given elsewhere (Gong, 1991). The observed apparent transverse velocity $v_{a p p}$ of a separation of a blob from the core of a quasar is related to the resultant velocity $V$ made of the velocity $v_{h}$ of Hubble flow and the velocity $u$ of local ejection through the expression,

$$
v_{a p p}=\frac{V \sin \alpha}{1+V \cos \alpha}, c=1
$$

where $\alpha$ is the angle between $V$ and $v_{h}$. Let the ejection velocity $u$ make an angle $\phi$ with $v_{h}$, then according to the formulae of the composition of velocity given by special relativity, we have the $V_{H}$ component of the resultant velocity $V$ in the $v_{h}$ direction,

$$
\begin{gathered}
V_{H}=\frac{v_{h}+u \cos \phi}{1+v_{h} u \cos \phi}, \\
V=\frac{\left\{\left(v_{h}^{2}+u^{2}+2 v_{h} u \cos \phi\right)-\left(v_{h} u \sin \phi\right)^{2}\right\}^{1 / 2}}{1+v_{h} u \cos \phi},
\end{gathered}
$$

where $v_{h}$ is uniquely given by the redshift $z_{q}$ of the quasar in question,

$$
v_{h}=\frac{\left(1+z_{q}\right)^{2}-1}{\left(1+z_{q}\right)^{2}+1}
$$

By solving the above equations, we can obtain values of $v_{h}, u, \phi, \alpha, V$ and $V_{H}$. By definition the Doppler factor of a moving source is given by,

$$
\delta=\frac{1}{1+\tilde{z}_{q}}=\frac{\left(1-V^{2}\right)^{1 / 2}}{1+V_{H}}=\frac{\nu_{m}}{\nu_{r}}=\frac{\lambda_{r}}{\lambda_{m}} .
$$

Subscripts $m$ and $r$ indicate, respectively, the frequency and the wavelength of the emission sources in motion and at rest. 
So far we have obtained the solution for the jet. By putting $u^{\prime}=u, \phi^{\prime}=\phi+180^{\circ}$ in the above equations, we can obtain values of corresponding quantities $\alpha^{\prime}, V^{\prime}, V_{H}^{\prime}$ and $\delta^{\prime}$ of the counterjet. From Eq.(5), the rest wavelength of the jet will be $\lambda_{, j}=\delta \lambda_{o b}$, where $\lambda_{o b}$ is the observed wavelength of the jet and the corresponding wavelength of the counterjot will be

$$
\lambda_{c j}=\lambda_{r j} / \delta^{\prime}=\delta \lambda_{o b} / \delta^{\prime}
$$

The wavelength of the counterjet $\lambda_{c j}$ is derived from three quantities, $\tilde{\sim}_{q}, \lambda_{c b}$ and $v_{a p p}$. Both $\tilde{\sim}_{q}$ and $\lambda_{o b}$ are observed quantities. $v_{a p p}$ is given by

$$
v_{\text {app }}=\mu D_{L} /\left(1+\tilde{z}_{q}\right) \propto D_{L},
$$

where $\mu$ is the observed proper motion. The luminosity distance is expressed by

$$
D_{L}=\left\{\tilde{z}_{q} q_{0}+\left(1-q_{0}\right)\left(1-\sqrt{2 q_{0} \tilde{z}_{q}+1}\right\} / H_{0} q_{0}^{2} \propto 1 / H_{0} .\right.
$$

Numerically $D_{L}$ changes very little when the deceleration parameter qu varies from 0.1 to 1.0, Thus, $\lambda_{c j}$ is mainly determined by the value of Hubble constant $H_{0}$. We, therefore, are alble to find out the value of $H_{0}$ reversely from the observed value of $\lambda_{c j}$.

\section{NUMERICAL RESULTS FOR SUPERLUMINALS AND SUBLUMINALS}

\subsection{Numerical Results for Superluminals $1928+738$ and $3 \mathrm{C179}$}

A few numerical results are quoted here from the paper (Gong, 1991) for illustration. For quasar $1928+738\left(v_{a p p}=7.0 c / h, z=0.302\right)$, the allowed values of the solution for $\phi$, the angle between the local ejection and the radial direction are in the range of $169^{\circ}-175^{\circ}$, the corresponding values of their speed, $u$, are in the range of $0.9940-0.9972 c$ and those of $\delta / \delta^{\prime}$ are in the range of $95-238$. For $3 \mathrm{C} 179\left(v_{a p p}=4.8 c / h, z=0.846\right)$, the corresponding values of $\phi, u$ and $\delta / \delta^{\prime}$ are, respectively, in the range of $168^{\circ}-176^{\circ}, 0.9943-0.9980 \mathrm{c}$ and $86-233$. As the value of $\delta / \delta^{\prime}$ is the ratio of the wavelength of the counterjet to $\lambda_{o b}$, the wavelength of the observed jet, obviously one is unable to observe the counterjet of superluminals with the wavelength as $\lambda_{o b}$.

As to the problem of the untolerable extension of the deprojected structure of some superluminal sources, we solve it satisfactorily. For quasar $1928+738$, the deprojected size given in Talble 1 of the paper by Simon et al. (1987) is $1090 \mathrm{kpc} / \mathrm{h}$, it reduces to $472-255$ $k p c / h$ if the ejection velocity decreases to $0.50-0.30 c$ with the ejection angle kept constant.

\subsection{Numerical Results of Subluminals M87 and $3 \mathrm{C84}$ and the Way for the Determination of Hubble constant $H_{0}$.}

We put $H_{0}=100 \mathrm{hkm} / \mathrm{s} / \mathrm{Mpc}$ with $h=1.0,0.75$ and 0.5 and proceed to compute the values of $\lambda_{c j}$ 's. U $\mathrm{U}_{p}$ to now we have found only two well-behaved subluminals with known redshift and proper motion as follows:
1) $\mathrm{M} 8 \mathrm{~T}=$ Virgo $\mathrm{A}=3 \mathrm{C} 274$ (Procas, 1987; Birett et al., 1988)
$\tilde{z}_{q}=0.004, \mu=1.1 \mathrm{mas} / \mathrm{yr}, v_{a p p}=0.21 \mathrm{c} / \mathrm{h}$;
2) 3C.84 = NGC1275 (Marr et al., 1988) 


$$
\tilde{\dot{\sim} q}=0.018, \mu=0.55 \mathrm{mas} / \mathrm{yr}, v_{a p p}=0.46 \mathrm{c} / \mathrm{h}
$$

The calculated values of various quantities solved for M87 and 3C.84 are tabulated separately. shown here only for $3 \mathrm{C} 84$ in Table 1 . From the tabulated values we plot $\lambda_{c j}$, the wavelength of the counterjet against $u^{\prime}$, the speed of local ejection, in Figure 1 for 3C84.

In the figure, these $\lambda_{c j}-u$ curves are located separately from each other. The top one is for $h=0.5$, the middle one for $h=0.75$ and the bottom one for $h=1.0$. On each curve there is a lowest point. i.e., the least ejection velocity $u_{\min }^{\prime}$. denoted by $\mathrm{B}, \mathrm{B}^{\prime}$ and $\mathrm{B}^{\prime \prime}$, respectively. on the top, middle and bottom curves. Points $L, L^{\prime}$ and $L^{\prime \prime}$ denote the longest expected wavelength of the counterjet for the respective cases of $h=0.5,0.75$ and 1.0. Similarly points $\mathrm{S}, \mathrm{S}^{\prime}$ and $\mathrm{S}^{\prime \prime}$ denote the shortest expected wavelength of the counterjet for three cases.

Table 1. Numerical Results for Subluminal 3C $84, \lambda_{o b}=1.3 \mathrm{~cm}$

\begin{tabular}{|c|c|c|c|c|c|c|c|}
\hline$V(c)$ & $V_{H}(c)$ & $u(c)$ & $\delta$ & $V_{H}^{\prime}(c)$ & $V^{\prime}(c)$ & $\delta^{\prime}$ & $\lambda_{c j}(\mathrm{cml})$ \\
\hline \multicolumn{8}{|c|}{$h=1.0$} \\
\hline 44078 & -.30196 & .45072 & 1.2859 & .33402 & .46083 & .66527 & 2.513 \\
\hline .41791 & -.17465 & .42429 & 1.1007 & .20901 & .43112 & .74631 & 1.917 \\
\hline .43482 & -.06555 & .43728 & .9637 & .10098 & .14031 & .81550 & 1.536 \\
\hline \multicolumn{8}{|c|}{$h=0.75$} \\
\hline .55148 & -.42991 & .56101 & 1.4455 & 45176 & .57074 & .56571 & 3.322 \\
\hline .52283 & -.27335 & .52793 & 1.1731 & .30603 & .53686 & .64598 & 2.361 \\
\hline .54213 & -.15114 & .54582 & .9899 & .18581 & .54986 & .7043 & 1.827 \\
\hline .60083 & -.20890 & .60145 & .8164 & .05651 & .60235 & .75554 & 1.409 \\
\hline \multicolumn{8}{|c|}{$h=0.5$} \\
\hline .70996 & -.61562 & .71757 & 1.8321 & .63729 & 72507 & .42062 & 5.663 \\
\hline .67706 & -.45841 & .68361 & 1.3588 & .48612 & .69019 & .48693 & 3.628 \\
\hline .69621 & -.33905 & .70075 & 1.0861 & .37024 & .70540 & .51729 & 2.729 \\
\hline .74914 & -.22245 & .75154 & .8519 & .25608 & .75408 & .52288 & 2.118 \\
\hline .84203 & -.08999 & .84264 & .5928 & .12525 & .84335 & .47755 & 1.614 \\
\hline
\end{tabular}

We are able to determine Hubble constant $H_{0}$ in the following way. The observed wavelength of the jet is $1.3 \mathrm{~cm}$. The longest expected wavelength are $5.64,3.32$ and 2.51 ('m, respectively for the cases $h=0.5,0.75$ and 1.0. Now, if the observed wavelength of the counterjet $\lambda_{c j}=4.0 \mathrm{~cm}$ is longer than those at $\mathrm{L}^{\prime}$ and $\mathrm{L}^{\prime \prime}$, then the value of $h$ must be less than 0.75 and lies between 0.5 and 0.75 . We put the new value of $h$ in the range $0.5 \sim 0.75$ and derive the new longest value of $\lambda_{c j}$ for the new value of $h$. The new one, say, $h_{m}$ which gives $\lambda_{c j}=4.0 \mathrm{~cm}$, is the upper bound of $h$ values for $3 \mathrm{C} .84$, i.e., $0.5<h<h_{m}<0.75$. Looking closely into the problem, one will find out $\lambda_{c j}$ is also effected by the direction of the ejection. The longer the wavelength $\lambda_{c j}$, the smaller the ejection angle between the radial direction and the ejection. Therefore, we need subluminals to make smaller angle with the radial direction. 
In the figure the wavelength $\lambda_{c j}$ for three curves overlaps each other on the left side. It is impossible to differentiate to which curve the observed $\lambda_{c j}$ belongs. However, the curves show that for each soluble value of $u^{\prime}$, there are always two solutions of $\lambda_{c j}$, one at the left side and the other at the right sicle of the lowest points $B, B^{\prime}$ and $B^{\prime \prime}$, respectively for earh of three curves. It can be shown that there is pretty chance to get the observed $\lambda_{c j}$ at the right side of $\mathrm{B}$ ( $\mathrm{B}^{\prime}$ or $\mathrm{B}^{\prime \prime}$ ), if more subluminals are observed.

\section{THE SOLUTION FOR THE OBSERVATION OF COUNTERJET OF GALAXY}

Arp et al. (1987a) claimed the ejection of a quasar from a galaxy as a result of the bipolar flow. As before we adopt a kinematic model involving twin processing jets in opposite direction. Thus, we can apply equations in Section 2 to solve the problem. Let $u$ be the velocity of the quasar ejected from a galaxy and $\phi$, the angle between $u$ and $v_{h}$, the velocity of Hubble flow, then Eq.(2) gives the component $V_{H}$ of the resultant velocity made of $u$ and $v_{h}$ in the direction of $v_{h}$. Eq.(5) can be rewritten as,

$$
V=\left\{1-\left(1+V_{H}\right)^{2} /\left(1+z_{q}\right)^{2}\right\}^{1 / 2}
$$

in which $\tilde{\sim}_{q}$, the redshift of the observed (quasar, is a known quantity. The value of $V_{H}$ derived from any value of $u \cos \phi$ in Eq.(2) will be allowalble solutions for the resultant velocity $V$ provided $V_{H} \leq \tilde{z} q$.

We apply the above method to two close associations of the galaxy and the quasar for illustration:

1) Markarian 205, $z_{q}=0.07 ; \mathrm{NGC} 4319, z_{g}=0.006$,

2) $3 \mathrm{C} .455, \quad z_{q}=0.543 ; \mathrm{NGC} 7413, z_{g}=0.0332$.

Both of them have been described by Arp et al., (1975) as the example of the ejection of (quasar by the nearby galaxy. The numerical results for the two examples are shown in Table 2 , in which values of $u$ and $\phi$ are, respectively, the speed and the ejection angle of the jet making with the radial direction so as to render the redshift of the ejecta equal to that of the observed quasar. Values of $z_{q}^{\prime}$ are the red (positive) and blue (negative) shift of the counterjet, a quasar in the present case. In case Mark.205 is ejected from NGC4319 in the process of bipolar flow, we should observe another quasar with one of the values $z_{q}^{\prime}$ in the opposite direction to Mark.205 with respect to NGC4319 as a center body. None of them has been observed. Neither for the case of 3C455 and NGC7413. Therefore, in the standpoint of kinematics and existing physical principles, both Mark.205 and 3C.455 are not ejected from their respective galaxy.

Table 2. Numerical Results for the Solution of Mark.205 and NGC4319 pair (Those for 3C455-NGC.7413 deleted due to space)

\begin{tabular}{rrrrrrrrr}
\hline$\phi\left({ }^{\circ}\right)$ & 0.000 & 43.7 & 71.6 & 79.1 & 86.0 & 100.1 & 124.5 & 136.9 \\
$u(c)$ & .615 & .0830 & .159 & .211 & .284 & .533 & .882 & .958 \\
$z_{q}^{\prime}$ & -.054 & -.0 .51 & -.032 & -.012 & +.028 & +.308 & +2.20 & +4.97 \\
\hline
\end{tabular}

The above conclusion for the two galaxy-quasar pairs might be extended to the problem of the ejection of quasars in the universe in general. Although an approaching source 


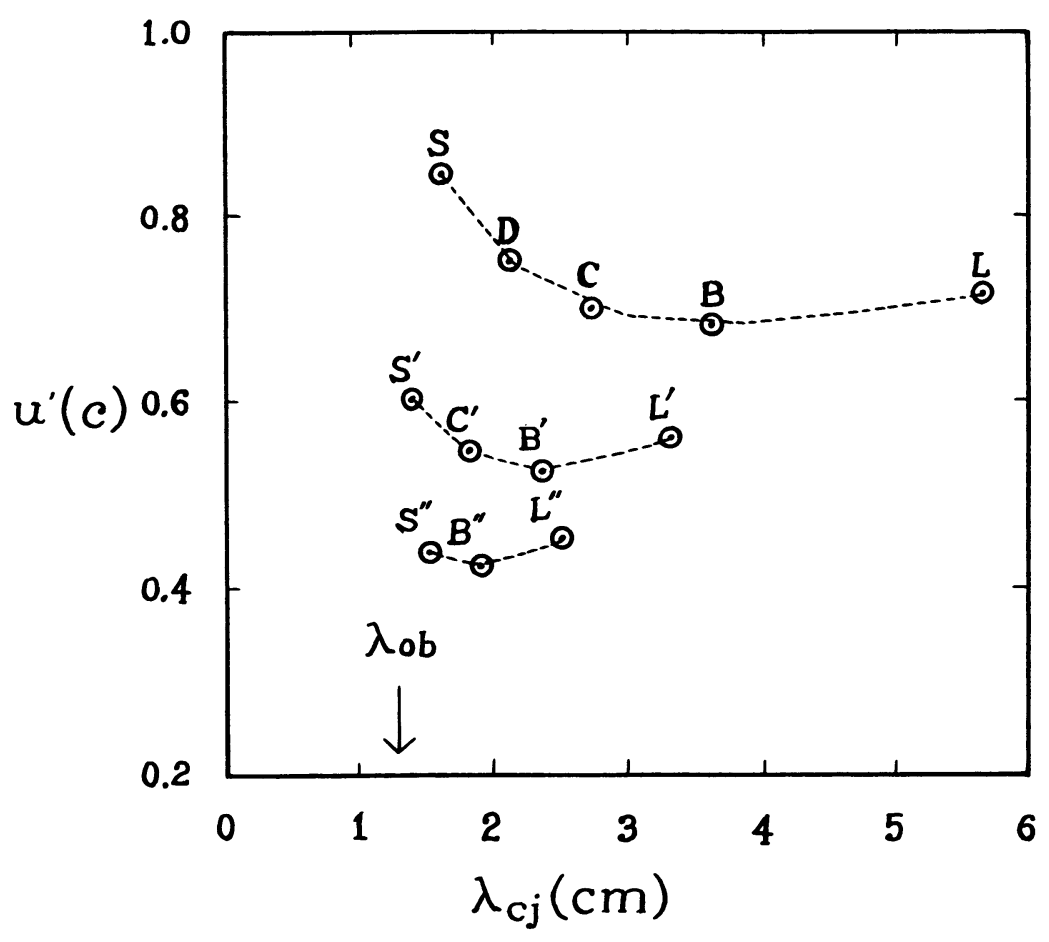

Fig.1. The expected wavelength $\lambda_{c j}$ of the counterjet vs the ejection velocity $u^{\prime}$ for subluminal $3 \mathrm{C} 84$. The top curve curve is for $h=0.5$, the middle one for $h=0.75$ and the bottom one for $h=1.0$. 
does not always show blue shift due to the time dilation factor, there are still quite a few approaching sources to have blue shift as shown in Table 2 . $U_{1}$ to now the number of the observed cuasars is over 4000 , none of them shows blue shift. Why? The only answer to it is that the quasar is not ejected from galaxy.

\section{REFERENCES}

Arp,H., Pratt, N. and Sulentic, J. 1975, Ap.J., 199, 565

Bahcall,J.N., et al., 1967, Ap.J., 149, L11

Belir,C., et al., 1976, A.J., 81, 147

Biretta,J.A. et al., 1988, in Impact of VLBI on Astrophysics and Geophysics, IAU Symp. 129, eds. Reid, M.J. \& Moran, J.M., 125

Biretta,J.A. and Owen,F.N., 1990, in Parsec-scale Radio Jet, eds. Zensus, J. A. \& Pearson, T.J., Cambridge Univ. Press, 37

Blandford,R.D. and Konigl,A., 1979, Ap.J., 232, 34

Dishon,G. and Weber,T.A., 1977, Ap.J., 212, 31

Gong,S.M., 1991, Ap. \& Space Sci., 175, 23

Horak,Z., 1978, Bull. Astro. Inst. Czech., 29, 126; 368

$\mathrm{Li}, \mathrm{Q}$, 1980, Acta Astron. Sinica, 21, 1

Marr,J.M. et al., 1988, in Impact of VLBI on Astroplysics and Geophysics, IAU Symp. 129, eds. Reid, M.J. \& Moran, J.M., 91

Pearson,T.J. and Zensus,J.A., 1987, in Superluminal Radio Sources, eds. Zensus, J.A. \& Pearson, T.J., Cambrialge Univ. Press, 1

Porcas.R.W., 1987, in Superluminal Radio Sources, eds. Zensus, J.A. \& Pearson, T.J., Cambridge Univ. Press, 12

Rees,M.J., 1966, Nature, 211, 468

Rees,M.J., 1967, M.N.,135,345

Simon,R.S. et al., 1987 in Superluminal Radio Sources, eds. Zensus, J.A. \& Pearson, T.J., Cambridge Univ. Press, 155

Sulentic,J.W. and Arp.H., 1987, Ap.J., 319, 687

Weymann, R.J. et al., 1977, Ap. J. 213, 619.

Wilkinson,P.N. et al., 1990, in Parsec-scale Radio Jet, eds. Zensus, J. A. \& Pearson, T.J., Cambridge Univ. Press, 152 